\title{
Reviewing the Ethical Dilemma of Surrogacy
}

\author{
Zheng Li \\ Philosophy College of Nankai University, Tianjin 300350, China \\ 1511515@mail.nankai.edu.cn
}

Keywords: Surrogacy, Ethical Dilemma, bioethics, feminism

\begin{abstract}
So far, new assisted reproductive technology (ART) involved surrogacy has raised a wide range of discussion in the world. Surrogacy, especially in some developed and developing countries, is the only way which can validly solve the problem of infertility rather than adoption. However, with the occurring of the new technology, lots of ethics problems made surrogacy a contested issue, obstructing the development of surrogacy while questioning the scholars and public: whether it is wrong to allow surrogacy by no means? Most ethical problem that have arisen out of surrogacy have been from bioethics, feminism to culture. This study reviews the findings of published papers regarding different aspects of surrogacy in China and some west countries. For example, first, some scholars in the aspects of feminism thought that the surrogate was demined by kinship, family, and Patriarchy. Surrogate has a tendency towards "objectification" by surrogacy agreements which aimed to "exploited women's body". Arguments from bioethics argue that the work of procreation cannot be regarded as an alienated work like other works, for is contains the respect of woman and the value of new birth. Besides the value and dignity of baby has been overlooked during the process. Last but not the least, surrogacy has raised different reflection among different culture and religion. Conclusion: both east and west countries exist a dilemma where resource are scarce while practical ethnological data.is not enough to support various theories. Scholars always put focus on one aspect or subject of surrogacy to illustrate the issue, making the issue captured into a trap of theoretical. Some fundamental principles should be taken into account the consideration of the availability of surrogacy. Lots of policies and laws should be taken into action to gain enough experience, data, and evidences to helping to examine the validity of theories.
\end{abstract}

\section{Introduction}

There is a subset of reproductive health care which assisted infertility included including treatments such as assisted reproductive technology (ART) and surrogacy [1]. Surrogacy is a new technology which empower surrogates, as a substitute, to accomplish the process of delivery of infants which partly have the genetic association between the surrogates and infants. Surrogacy, based on the source of sperm and egg, involved full surrogacy (host surrogacy) and partial surrogacy (straight surrogacy). The sperm used in full surrogacy was originated from intended father while the egg was originated from the surrogate. The egg and sperm used in partial surrogacy, on the other hand, are originated from the donors or the surrogates. Given the fact that partial surrogacy has caused a wide range of controversial arguments between scholars and it is obviously complex and difficult to talk about, my ethics concentration limited by full surrogacy mainly on the dilemma of full surrogacy on the ethics perspective.

For decades, surrogacy had raised lots of complex legal and ethical problem. Debates about surrogacy was began with the case afterbirth of Baby M in the United States in 1986. Lawmakers are questioned the effectiveness of payment for making or engaging a surrogacy arrangement and the legal status of two "mother". However, less than one-third of the proposed laws have clear provisions establishing the legal parents after the birth of a baby conceived pursuant to a surrogate agreement. Under the remaining proposals, recourse to the courts is still the only way for the biological father or biological mother to gain legal custody of the child when conflict happened ${ }^{[2]}$. The Department of Health \& Social Security of British established Committee of Inquiry into Human Fertilization and Embryology in 1982 which dedicated to" examine the social, ethical and legal implications of recent, 
and potential developments in the field of human assisted reproduction ${ }^{[3]}$." This committee published an influential report called "REPORT OF THE COMMITTEE OF INQUIRY INTOHUMAN FERTILISATION AND EMBRYOLOGY". Analyzing several current new assisted reproductive technologies included surrogacy, the report argued that not only the risk of danger surrogacy conceived was far behind the interest it could acquire, but also is unacceptable in ethics or morality ${ }^{[4]}$. Under such circumstance mentioned that both non-profit and profit organizations making surrogacy service could lead to the encouragement of the growth of surrogacy, the committee strongly recommended that the legislation should "render criminal the creation or the operation in the United Kingdom of agencies whose purposes include the recruitment of women for surrogate pregnancy or making arrangements for individuals or couples who wish to utilize the services of a carrying mother ${ }^{[5]}$,

\section{Arguments Against Surrogacy}

Arguments against surrogacy in the field of ethic could, at some point, be classified into three group based on distinctive perspectives. Therefore, lawmakers always must face a cascade of problematic critique by public, medical field, ethicists, philosopher, feminists, etc.

\subsection{Bioethics}

Bioethics make combination of human dignity and autonomy, researching the contradiction of those two issues. Through the driving of market law, surrogates could make profit by selling their physically capability of breeding and wombs independently without the invading of other forces. Those behaviors, on the other hand, could be regarded as a tendency towards the objectification of women. Surrogates, themselves, could have possibility to become a tool functioning to achieve goal not the goal itself. It could not be overlooked that some commercial surrogacy occurred in Asian such as India make the "mother-worker" duality controlled by "recruitment of guilt-ridden mothers to disciplining of poor, rural, uneducated Indian women ${ }^{[6]}$ ". Conflicts often produced between the identity of a mother artificially regulated and a pure mother-worker.

In addition, surrogacy could also have influence on the autonomy of infant, which has leaded to a crucial ethic concerns. Given the fact that surrogacy was established on the arrangement and contracts between intended parents and surrogates, at some point babies could be regarded as products "bought" from his surrogate (or biological mother), thus ignoring intrinsic value of themselves and the natural intimate relationship with their biologic mother. For example, Clara Watson notes that surrogacy, by its nature, necessarily undermines the human dignity of both the woman and child born through such arrangements. Some scholars also put attention on India, where international surrogacy was allowed and under most comprehensive legal protections [7]. But the right and the claim of child has less been concerned. First, it is virtually uncontested that a child would live better when raised with its biological parents. In addition, the psychosocial problems of child who is the result of a surrogates has not clear. Many concerns rested on if the self-identity of a child would be affect when he or she knows there was a law mother and a biological mother who gave birth to him or her unless in the condition that the surrogate was anonymous from the very beginning.

\subsection{Feminism}

Those assisted reproductive technology (ART) are also subjected to critiques from feminism, wherever they did. From feminists' point of view, procreation is regarded as a paramount obligation of women who are closely attached by a sense of bringing forth offspring. After industrial Revolution in 18th century, women were required to stay home in terms of breeding babies and raising children while man had to work in factories. By means of this distinctive difference which suggested the fountainhead from which built the increasingly sexual inequality. It is reasonable that some moral issues that whether women have right to abortion was been discussed for centuries. For instance, feminists argue that it is the public due to some intents refuse to render women the choice to freely on the procreation, controlling women to become imperfect. We can conclude that women are powerless not only on the right to select procreation but also the results of breeding, accelerating passiveness entrusted to women. There is no wonder that many radical feminists describe surrogacy a way to reducing women to "uterine environments," "living laboratories," "test-tube women," 
"mother-machines," "fetal containers ${ }^{[8] "}$ and "vessels. And argue that reproductive technologies exploit women's body.

Surrogacy is a life-changing process to surrogates. Some scholars have substantiated this conception to make it more than theoretical. For example, Jie Yang studied the ambivalence of surrogate herself, moral and emotional claims by mentioning the experiences of her interviews talking their surrogacy process in person, pointing out that by means of informal surrogacy, a woman's womb is communal, not private ${ }^{[9]}$. And a long-term emotional and psychological distress also happens simultaneously when stretch marks remained and relinquish a child, related to the child or sometimes the intended father through sexual relationships ${ }^{[10]}$. Under a society of paternity, a surrogate's emotional disease and contribution, likely, been hidden behind the enjoyment of the families. Those surrogates experience again stressed their body's permeable boundaries. In that regard, many feminists' critiques often describe the process of surrogacy of women "objectification", that is, a denial of the mother-child natural gestational bond.

\subsection{Culture, Religion}

Tradition opinions state on the following moral communities, which hold different defines and value judgment on the surrogacy issue. Moreover, different moral communities have distinguished culture and religions which have deep influence on behaviors and opinions of communities, making the problem whether it is wrong to surrogacy more complex. From the perspective of tradition culture, family is the basic cell organized the world. By means of falling love, intercourse, conceiving a child, gestation, and delivery a baby, new life is born and fasten the connection between the couple. Surrogacy, however, comes to invade and, at some point, reconstruct the whole conception of family by evilly introducing into a "third party"[11]. For example, in China, some scholars quoted that marriage might not contain love, but cannot produce families and baby. Separating love from procreation is unacceptable. Several cultures in Asian, "Producing offspring" was regard as a paramount duty of marriage, as an old Chinese saying goes:" There are three remarks implying you are not filial piety, most serious of which is having no offspring ${ }^{[12]}$. Susan Brownell also noted that Chinese people find themselves caught in "a web of interdependencies" which reflect the importance and acute of family, state, and body ${ }^{[13]}$. Body has been changed into permeable, public, and it even has a directly connection with the land, the ancestors. Other culture and religions also share similar character. For example, most Christian churches forbid surrogacy, because of the contrary to the unity of marriage and the dignity of procreation of a human being ${ }^{[1} 4^{1}$. Some theologist such as Ramsey also accuse this kind of process of procreation of exclude procreation from love, which distorting the sacred meaning of procreation ${ }^{[1} 5^{]}$.

\section{Conclusion}

During the period of postindustrial, there must be many factors that boost the resurgence of surrogacy. Research should not only an interdisciplinary but also a complex problem. However, both east and west countries exist a dilemma where resource are scarce while practical ethnological data is not enough to support various theories. Scholars always put focus on one aspect or subject of surrogacy to illustrate the issue, making the issue captured into a trap of theoretical.

From my perspective, some fundamental principles should be considered the consideration of the availability of surrogacy. First, any single point of research cannot determine the application of surrogacy. A paramount reason caused the dilemma of surrogacy is rested on the condition where many main roles is played during the whole process of surrogacy: intended parents, surrogate baby, sometimes agent, intermediaries, legislative, government, and public. Different interest, different seeker. Considering the fact how crucial impact have on each of roles' interests involved is highly of importance and subsequently take them in turn based on importance. Second, though surrogacy has been forced to the interrogates from many aspects, it could also receive many proponents arguing surrogacy can be applied into assisted reproduction. Philosopher Engelhardt, specializing in philosophy of medicine, said surrogates, as a moral subject, willing to use his body according the arrangement her had set, is equivalent to that of hiring other jobs such as ballet dancer, singer, for there is nothing implying exploiting body between both, contrary to the proclaim radical feminist 
mentioned. Because both are using some capabilities of her body to bring happiness and enjoyment for others. But his opinion was reject by Anton van Niekerk and Liezl van Zyl, who differentiate between surrogacy, prostitution, and other jobs. They said that it is women's reproductive labor that should be afforded special respect that is degraded by means of "alienated labor" (that is, according to Hegelian sense, a product is separated from its producer and producer surrendered it to someone else. ${ }^{[1} 6^{]}$, which have strong distinctive difference from other jobs. In other ground, Elly Teman called "explicit self-objectification of the body", which radical feminist repeat to argue that reproductive technologies exploit women. Claims that women are implanted with seeds of the patriarchy and lacking control over body, is refuted by surrogates themselves. While some of surrogates regard its body as an oven that "bakes the bread for hungry people" to help others. In addition, the problem that relinquishing baby scarcely when the child is delivered can be reluctant for woman to cut down the intimate relationship between biological mother and child also subsequently cause traumatic physically and psychologically also is demonstrated rare., according to many survey estimated in the late-1990's [17].

But even so, lots of policies and laws should be taken into action. Some countries attempted to let surrogacy reconciled with the actual cultural environment. For example, according to a proposal for legislation passed by Israeli parliament in 1996, the surrogate mother should be single or divorced, otherwise the child is 'illegitimate' according to the Jewish religion. To make the religion of child equal to that of its mother, the surrogate mother should be of the same of the intended parents. Similarly, some attempts were applied to transform the bodily sensations of gestation and pregnancy conceived by surrogate to the intended mother for enabling the intended mother to construct pregnant identity to filling the gap of identity difference. Majority of details should be taken into consideration. And the total application of full surrogacy is still a long way off.

\section{References}

[1]. Saravanan S. An ethno methodological approach to examine exploitation in the context of capacity, trust, and experience of commercial surrogacy in India [J]. Philosophy, Ethics, and Humanities in Medicine, 2013, 8(1):1-12.

[2]. Andrews L B. The aftermath of Baby M: proposed state laws on surrogate motherhood. [J]. Hastings Center Report, 1987, 17(5):31-40.

[3]. Security D O H, Warnock M. Report of the Committee of Inquiry into human fertilization and embryology [J]. Irish Nursing News, 1984, 5(3):7.

[4]. Pan R H. The Historical Observation of Regulations on Surrogate Pregnancy and Its Reforming in the United Kingdom [J]. Medicine \& Philosophy, 2006.

[5]. Security D O H, Warnock M. Report of the Committee of Inquiry into human fertilization and embryology [J]. Irish Nursing News, 1984, 5(3):7.

[6]. Paned A. Commercial Surrogacy in India: Manufacturing a Perfect Mother - Worker [J]. Signs: Journal of Women in Culture and Society, 2010, 35(Volume 35, Number 4):969-992

[7]. Vinita Singh • Pushpawati Thakur - Byasdev • Anjum Assistant. DOES SURROGACY INVOLVE MAKING FAMILIES OR SELLING BABIES? [J]. International Journal of Healthcare Sciences (Online)Vol. 2, Issue 1, pp: (129-130), Month: April 2014 -September 2014

[8]. Leissner O. Elly Teman, Birthing a Mother: The Surrogate Body and the Pregnant Self [J]. Nashim A Journal of Jewish Womens Studies \& Gender Issues, 2011, 5(21):193-198.

[9]. Yang J. Informal Surrogacy in China [J]. Body \& Society, 2014, 21(1):999-1009.

[10]. Anleu S L R. Reinforcing Gender Norms: Commercial and Altruistic Surrogacy [J]. Acta Sociologica, 1990, 33(1):63-74.

[11]. Englelhardt HT. The Foundations of Bioethics, 2d. edn [M] New York: Oxford University Press, 1996.

[12]. Yukuan Niu. The Ethics of the Surrogacy Issue Thinking [D] Southwest University of Political Science \& Law; SWUPL., 2013.

[13]. Susan Brownell. Training the body for China: [M]. University of Chicago Press, 1995. 
[14]. Benshushan A, Schenker J G. Legitimizing surrogacy in Israel [J]. Human Reproduction, 1997, 12(8):1832-4.

[15]. Ramsey P. Fabricated man: The ethics of genetic control [J]. 1970.

[16]. Saravanan S. An ethnomethodological approach to examine exploitation in the context of capacity, trust, and experience of commercial surrogacy in India [J]. Philosophy, Ethics, and Humanities in Medicine, 2013, 8(1):1-12.

[17]. According to Elly Teman, Lori Andrews (The Clone Age [New York: Henry Holt, 1999]) estimates that less than 1 percent of surrogates have changes heart, while others suggest figures as low as 0.25 percent (Chris Taylor, “One Baby Too Many,” Time, August 27,2001. 